\title{
Timing analysis of 10-day long BeppoSAX observation of GRS $1915+105$ in its $\rho$ class
}

\section{E. Massaro}

Department of Physics, 'Sapienza University', Roma, Italy

E-mail: enrico.massaro@uniroma1.it

\section{G. Ventura}

Stazione Astronomica di Vallinfreda, Italy

\section{F. Massa}

Stazione Astronomica di Vallinfreda and INFN-Sezione di Romal (retired), Italy

\section{T. Mineo, G. Cusumano}

INAF-IASFPa, Sezione di Palermo, Italy

\section{P. Casella}

Astronomical Institute, University of Amsterdam, The Netherlands

\section{T. Belloni}

INAF-OAB, Merate, Italy

\section{Feroci}

INAF-IASF, Sezione di Roma, Italy

The microquasar GRS 1915+105 was observed several times by BeppoSAX. Here we present an extensive timing analysis of the longest observation, performed on October 2000. During this 10-day long observation the source was mainly observed in its $\rho$ class, characterized by series of pulses, with recurrence times varying between 40 and 90 seconds. The time profile of the pulses is characterized by a rather smooth rising branch followed by one or more peaks with a fast decline. We apply different methods to study the time structure of the X-ray light curve, and describe the statistical properties of the observed variability.

VII Microquasar Workshop: Microquasars and Beyond September 1-5 2008

Foca, Izmir, Turkey 


\section{Introduction}

GRS 1915+105 was observed by the Narrow Field Instruments onboard the BeppoSAX satellite on several occasions. The amount of data collected in these pointings is very large and requires a long and complex analysis.

In this contribution we present preliminary results on some particular aspects of the time behaviour of GRS 1915+105 observed in the course of last and long pointing in October 2000. The source was for several days in the $\rho$ class described by Belloni et al. (2000) [1], characterised by series of flares, with a variable recurrence time between 40 and 90 seconds.

\section{Light curves, Fourier periodograms and wavelet spectra}

Light curves were obtained in the energy bands of two detectors: MECS (1.6-10 keV) and PDS (15-100 keV). We obtained 108 well sampled time series spanning over a time interval of about 600,000 seconds; 87 of which, with durations ranging from $\sim 1,000$ to more than 3,000 seconds, were used in our analysis. Fig. 1 (left panel) shows three segments of MECS light curves that in the following are used as examples to describe the behaviour of GRS 1915+105. The source showed the rather regular behaviour characteristic of the $\rho$ class of [1]; the mid segment, however, reveals an intermediate behaviour between this class and the $\kappa$ one. The Fourier periodgrams of the same series are shown in the central panel, and the wavelet spectra obtained using a Morlet 12 wavelet [2] are plotted in the right panel.

We found that some Fourier periodgrams show a single dominant peak indicating a stable recurrence time, $T_{\text {rec }}$, between subsequent flares, whereas other spectra have two or more dominant features (excluding the harmonics of the main peak). We introduced thus a first classification of the time series based on the dominance of the highest peak: ' $S$ ' type either when a single peak is largely prominent over the background or when the ratio between the powers of the highest peak to the second one is greater than 3; ' $\mathrm{D}$ ' type when two peaks are apparent and their power ratio is lower than 3; 'M' type when the power is distributed among three or more peaks. S series are the most frequent, occurring in $47 \%$ of cases; the $34 \%$ of series are of M type, and the $14 \%$ are of D type.

A more complete description of the source behaviour can be obtained from wavelet spectra (see Fig. 1, right panel). S and D series are generally characterised by an uninterrupted and nearly constant strip, centred at the $T_{r e c}$ value. The poor resolution of wavelet compared to periodgrams does not allow to distinguish clearly the peak separation in the $\mathrm{D}$ series that is typically of a few seconds. Changes of $T_{r e c}$ are present in the wavelet spectra of these series, but their duration is limited to a few cycles. M series shows typically much more irregular patterns also over short time intervals. Power is distributed in a rather broad strip that shows meanderings, bifurcations and interruptions corresponding to the simultaneous occurrence of short and long recurrence time intervals between subsequent bursts.

We improved the above classification by adding three new subclass types based on the wavelet spectra: "s" (low case) type when the power is distributed along a single continuous linear belt, "o" type when this belt has small amplitude oscillating behaviour, and "i" type when it appears 

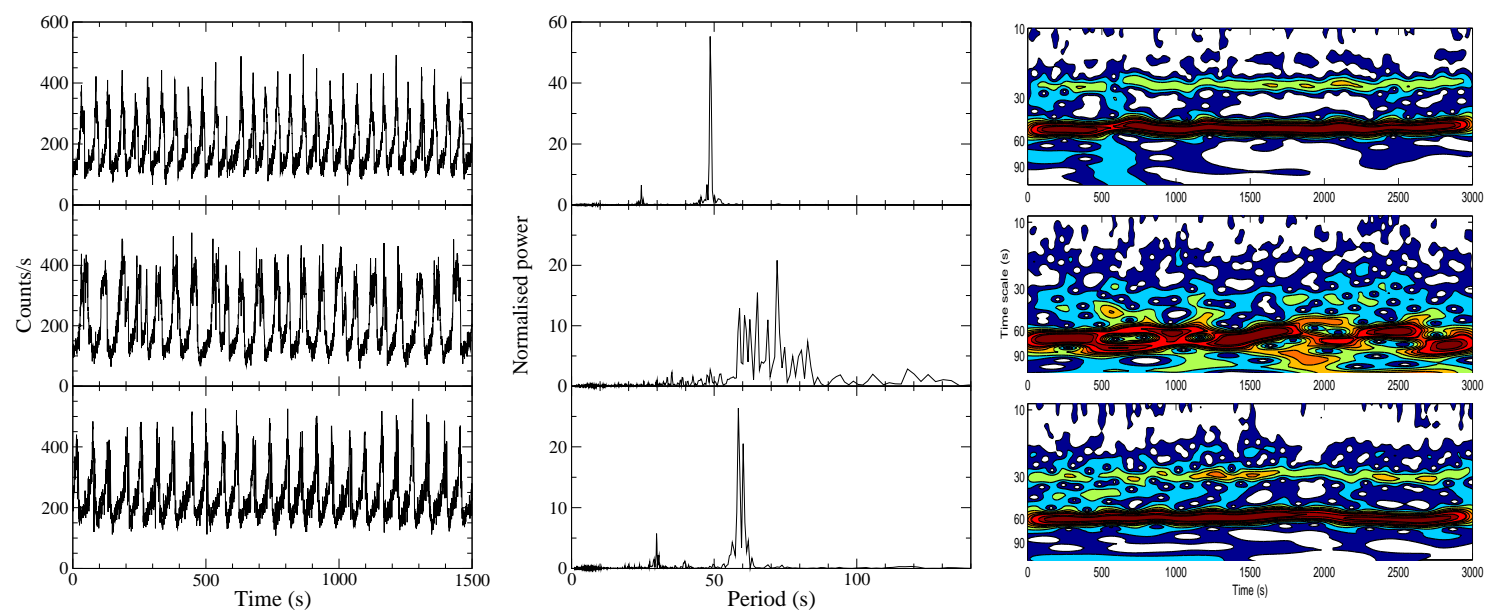

Figure 1: Left panel: Three segments of MECS (1.6-10 keV) light curves during the long BeppoSAX observation of October 2000. Time bin width is 0.5 seconds. The top and bottom curves show the typical time pattern of the $\rho$ class, while the central one has a more complex structure. Central panel: Periodgrams of the same three MECS time series classified (top to bottom) as S type, M type and D type. Right panel: Wavelet spectra of the same three MECS series.

irregularly discontinuos. The majority of S spectra are Ss type, but few of them are $\mathrm{Si}$; conversely, $\mathrm{M}$ spectra are mostly Mo or Mi, and only 3 are Ms.

On the basis of this classification we distinguished two modes of the behaviour of GRS $1915+105$ in the $\rho$ class: a regular mode, essentially corresponding to Ss/So or Ds/Do series, and an irregular mode, corresponding to i (mostly Mi) type wavelet spectra. In the first part of the pointing the source exhibited a regular mode, then it changed to the irregular one to return to be regular in the last part. Each of these phases occurred on a time scale having a typical duration of one or two days. D series are found in both modes and can be considered as a transition between $\mathrm{S}$ and $\mathrm{M}$ types. S spectra occasionally observed in sequences of $\mathrm{D}$ or $\mathrm{M}$ spectra are of type $\mathrm{Si}$, indicating that the behaviour of GRS 1915+105 remained irregular, despite the power appears in a single peak.

\section{3. $T_{r e c}$ - count rate correlation}

An interesting result is the long term change of $T_{r e c}$. It was around $45-50 \mathrm{~s}$ at the beginning of the pointing, and increased up to more than $80 \mathrm{~s}$ in the last time series. Despite the large scatter, an increasing trend is well apparent from the left panel of Fig. 2: a linear best fit of the values of $S$ spectra gives a linear correlation coefficient $r=0.78$ and an increasing rate of $T_{\text {rec }}$ of about 3 s/day.

It is interesting to note that the mean count rate increases during the pointing from $\sim 200$ counts/s to $\sim 250$ counts/s. Fig. 2 (right panel) shows the positive correlation between $T_{\text {rec }}$ and the mean count rate: in this case we found $r=0.92$ (using only data of S series and excluding Si series); the resulting increase of $T_{\text {rec }}$ is about 4 seconds for a count rate change of 10 counts, that corresponds approximately to a brightening of $5 \%$. We found that the long term brightening is 

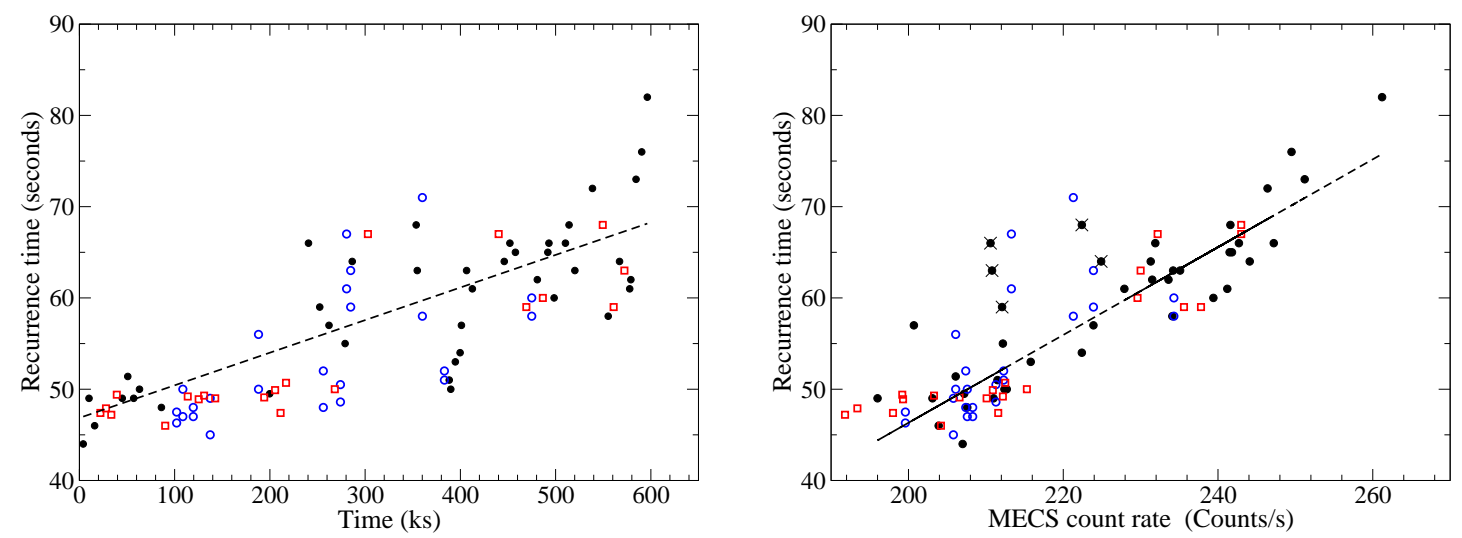

Figure 2: Left panel: The evolution of $T_{\text {rec }}$ of MECS series in the course of the observation. In the abscissa is the time elapsed from the beginning of the pointing; symbols correspond to S (filled circles), D (open circles) and M (open square) series. For the D series the times of both peaks are plotted, while the points of M series correspond to the centroid of interval where peaks are present. Right panel: the relation between $T_{\text {rec }}$ and the mean count rate in the MECS band. Dashed line is a linear best fit to $\mathrm{S}$ series only after the exclusion of Si series (crosses).

related to the baseline level, whereas the flares' mean amplitude ramained approximately constant. This result is not in contrast with the finding of Naik et al. (2002) [3], who noticed that the burst strength decreases for increasing $T_{r e c}$. These authors, in fact, define the burst strength as the ratio of the peak count rate to lowest level, that when increasing produces the burst strength lowering, as it appears in Fig. 1 of their paper [3].

\section{Analysis of flares' structure}

As pointed out by [1], the structure of individual flares, when analysed with a high time resolution, shows several subflares, fast spikes and dips having durations down to a few hundreds of milliseconds, and likely even shorter.

To study the mean profile of flares, we used a statistical approach and analysed the flare shapes, after running average smoothing with a window of 5.5 seconds. We defined a "multiplicity" number, corresponding to how many subflares can be distinguished. (Fig. 3, left panel): the large majority of flares (87\%) have multiplicity one $(\mathrm{m} 1)$ or two $(\mathrm{m} 2), \sim 9 \%$ are of $m 3$ type, and only the remaining $\sim 4 \%$ belongs to $m 4$ and $m 5$ types. Moreover, $m 3$ to $m 5$ flares are frequent in Mi series, whereas Ss series contain $m 1$ and $m 2$ flares, independently of the value of $T_{\text {rec }}$.

As it appears in the left panel of Fig. 3, the typical length of flares increases with the multiplicity. In the right panel of Fig. 3 we plotted the mean time in which the maxima of flares occur, $T_{\max }$, vs the average $T_{r e c}$ for each multiplicity type in the first half of the observation (47 data series). Points are very well aligned, indicating that the mean recurrence time of flares is clearly related to the time necessary to reach the maximum (and viceversa). 

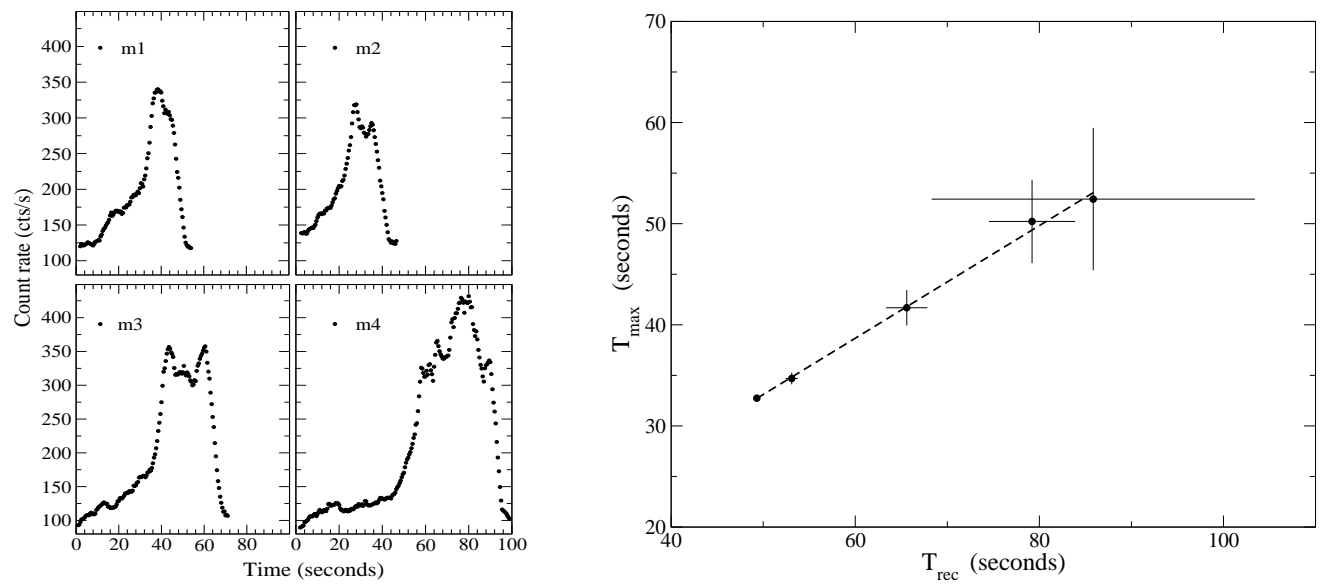

Figure 3: Left panel: examples of multiplicity types ( $m 1$ to $m 4$ ) of flares. Right panel: plot of the mean $T_{\max }$ vs $T_{\text {rec }}$ for the various multiplicity types (points corresponding to $m 1$ to $m 5$ are in the order from left to right).

\section{Summary}

The main results of our analysis of the long BeppoSAX pointing of GRS 1915+105 performed in October 2000 can be summarized as follows:

1) the source was observed for several days in the $\rho$ class and showed phases of regular variability, characterised by a stable $T_{r e c}$, producing a smooth feature in the wavelet spectrum, in alternation with irregular phases, showing large variations of the flares' structure and $T_{\text {rec }}$;

2) the average $T_{\text {rec }}$ varied in the course of the observations, with a positive correlation with the mean count rate, indicating the variations were slower when the source was brighter;

3) flares are structured in sub-flares and show time scales that vary linearly with $T_{\text {rec }}$.

Time series consisting in sequences of flares, as those typical of the $\rho$ class, can be the consequence of thermal-viscous instabilities in an accretion disk as shown by Taam \& Lin [4] and Watarai \& Mineshige [5]. It is important to investigate which are the physical conditions for a disk for reproducing the various observed time scales and their relations with the disk luminosity as derived from the present analysis.

\section{References}

[1] T. Belloni, M. Klein-Wolt, M. Méndez, et al. 2000, A\&A 355, 271

[2] C. Torrence, G.P. Compo 1998, BAMS 79, 61

[3] S. Naik, et al., 2002, MNRAS, 330, 487

[4] R.E. Tamm, D.N.C. Lin 1984, ApJ 287, 761

[5] K.-Y. Watarai, S. Mineshige 2003, ApJ 596, 421 